\title{
Surgical indication of iNPH (idiopathic normal pressure hydrocephalus)
}

\author{
Teruo Kimura*, Toshihide Sugimura, Shin Fukuda, Makoto Miyano, Masaaki Hashimoto \\ From Hydrocephalus 2015 \\ Banff, Canada. 18-21 September 2015
}

\section{Introduction}

Some patients with iNPH often can recover independent $\mathrm{AD}$ and can be easily rehabilitated after shunt surgery. However, other patients sometimes cannot recover independent ADL even if improvement is obtained after treatment, because of some factors. The purpose of this study is to examine the influence of the factor determining the benefit from shunt surgery.

\section{Methods}

All patients underwent a tap test during 9 years from 2002 to 2010 , and 154 probable iNPH patients who showed a clinical improvement of at least $10 \%$ underwent shunt placement (V-P shunt, 12 patients; L-P shunt, 142 patients). One hundred and thirty-three patients who could be followed up for one year were investigated for age, the interval from onset to surgery, severity, degree of improvement by tap test, MRI findings (DESH or non-DESH).

\section{Results}

According to older patients, the postoperative recovery of mRS, gait disturbance (GD), dysuria, and cognitive impairment $(\mathrm{CI})$ was poor. According to long interval from onset to surgery (group $1<2<3<4<5$ ), the score (mRS, iNPHGS) of them showed higher points mean more severity. There were correlations between the length of the interval from onset to surgery and severity $(P<0.01)$, between the length of the interval from onset to surgery and the degree of improvement after surgery $(\mathrm{P}<0.01)$, and between severity and the degree of improvement after shunt $(\mathrm{P}<0.01)$. There were correlations between severity of GD $(P<0.01)$ and the degree of improvement after surgery. In tap test, the patient (group A) of remarkable recovery of postoperative score, the symptom was improved, especially, mRS, GD significantly $(\mathrm{P}<0.01)$, keeping good $\mathrm{ADL}$ after one year $(\mathrm{p}<0.01)$. The patient with DESH in preoperative MRI findings (coronal view) was more improvement than patients with non-DESH in mRS, GD, dysuria and CI, significantly $(\mathrm{p}<0.01)$, keeping good independent ADL after one year postoperatively.

\section{Conclusions}

Surgical indication is decided with careful consideration of social indication including the comorbidity, fully informed consent should be made.

\section{Published: 18 September 2015}

\section{References}

1. In vivo single-voxel proton MR spectroscopy in brain lesions with ringlike enhancement. NMR Biomed 2001, 14(6):339-49.

2. Effect of mild hypothermia on energy state recovery following transient forebrain ischemia in the gerbil. Exp Brain Res 2002, 1(2):121-4.

3. Diagnosis and treatment of progressive space-occupying radiation necrosis following stereotactic radiosurgery for brain metastasis: value of proton magnetic resonance spectroscopy. Acta Neurochir (Wien) 2003, 145(7):557-64.

4. Evaluation of the response of metastatic brain tumors to stereotactic radiosurgery by proton magnetic resonance spectroscopy, 201TIC1 single-photon emission computerized tomography, and gadoliniumenhanced magnetic resonance imaging. J Neurosurg 2004, 100:835-841.

5. Multiple basilar artery trunk aneurysms associated with fibromuscular dysplasia. Acta Neurochir (Wien) 2004, 146:79-81.

6. Increase in glutamate as a sensitive indicator of extracellular matrix integrity in peritumoral edema: a 3.0-tesla proton magnetic resonance spectroscopy study. J Neurosurg 2007, 106:609-613.

\section{doi:10.1186/2045-8118-12-S1-P25}

Cite this article as: Kimura et al:: Surgical indication of iNPH (idiopathic normal pressure hydrocephalus). Fluids and Barriers of the CNS 201512 (Suppl 1):P25.

* Correspondence: tekimura-nsu@umin.ac.jp

Dohtoh Neurosurgical Hospital, Okhotsk Cerebrovascular Center, Japan 